\title{
Solar fuels editorial $\dagger$
}

\author{
Cite this: Chem. Soc. Rev., 2013, \\ Siddharth Dasgupta, Bruce S. Brunschwig, Jay R. Winkler and Harry B. Gray
}

42, 2213

DOI: $10.1039 /$ c3cs90016a

www.rsc.org/csr

Every major change in the living standards for humans on our planet has had an energy revolution at its heart - the advent of the industrial age with the steam engine and use of coal, the internal combustion engine and large-scale electricity generation. The energy demand, primarily from emerging economies, will double by 2050. The countervailing urgency of the threat of climate change requires a major shift in our energy sourcing, creating four new trends that will shape the current century: electrification, decarbonization, localization, and optimization.

One of the "holy grails" of 21st century science is the economical conversion of solar energy into chemical fuels. Research targeting efficient utilization of solar energy is inherently interdisciplinary, involving inorganic and organic synthesis, solid state chemistry and physics, electrochemistry, chemical kinetics and mechanism, and theoretical and computational chemistry. Solar fuels production involves three basic components: light absorption; charge transport; and multielectron redox catalysis. This themed issue brings together some of the world's foremost experts to discuss current research and future prospects in this area.

In "Long-lived charge separated states in nanostructured semiconductor photoelectrodes for the production of solar fuels", Cowan and Durrant

California Institute of Technology, Pasadena,

California 91125, USA

$\dagger$ Part of the solar fuels themed issue.
(DOI: 10.1039/C2CS35305A) discuss designs to increase charge carrier lifetimes in semiconductor photoelectrodes for water photolysis and carbon dioxide reduction that are based on lessons learned from natural photosynthesis. A key consideration is the design of interfaces that achieve a sufficient increase in charge carrier lifetime with a high quantum yield, whilst minimizing the energy loss associated with this lifetime gain.

Osterloh (DOI: 10.1039/C2CS35266D) reviews the current state of research on nanoscale-enhanced photoelectrodes and photocatalysts in "Inorganic nanostructures for photoelectrochemical and photocatalytic water splitting". The effects of nanostructuring on carrier generation and collection, multiple exciton generation, and quantum confinement, as well as implications of particle size on surface recombination, the size of space charge layers, and on the possibility of controlling nanostructure energetics via potential determining ions, are described.

In "Nanostructured photoelectrodes based on $\mathrm{WO}_{3}$ : applications to photooxidation of aqueous electrolytes", Bignozzi, Caramori, Cristino, Argazzi, Meda and Tacca (DOI: 10.1039/ C2CS35373C) focus on the preparation and modification of nanostructured $\mathrm{WO}_{3}$ thin films for photoanodes. $\mathrm{WO}_{3}$ is one of the few materials that can achieve efficient water photo-oxidation under visible illumination, stably operating under strongly oxidizing conditions; achieving almost quantitative photon to electron conversion.
Park, McDonald and Choi (DOI: 10.1039/C2CS35260E) report on "Progress in bismuth vanadate photoanodes for use in solar water oxidation". Latest efforts to improve performance include morphology control, formation of composite structures, composition tuning, and coupling of oxygen evolution catalysts, and are directly applicable to the understanding and improvement of other photoelectrode systems.

Artero and Fontecave (DOI: 10.1039/ C2CS35334B) give us their perspective on "Solar fuels generation and molecular systems: is it homogeneous or heterogeneous catalysis?" While allowing for fine tuning of catalytic properties through ligand design, molecular approaches are frequently criticized because of the inherent fragility of the resulting catalysts when exposed to extreme redox potentials. In a number of cases the true catalytic species is heterogeneous arising from the transformation of the initial molecular pre-catalyst. In their review, they discuss cases in which this issue has been directly addressed, similar to homogeneous hydrogenation reactions in organometallic chemistry.

In "Design and development of photoanodes for water-splitting dye-sensitized photoelectrochemical cells", Swierk and Mallouk (DOI: 10.1039/C2CS35246J) discuss dye sensitized solar cells (DSSCs) that use low-cost materials, feature tunable molecular sensitizers, and exhibit quantum efficiencies near unity. These features can be exploited by functionalizing DSSCs with catalysts for water oxidation and reduction. 
Parent, Crabtree and Brudvig (DOI: 10.1039/C2CS35225G) compare chemical oxidants for driving water-oxidation catalysts, focusing on the advantages and disadvantages of each in "Comparison of primary oxidants for water-oxidation catalysis".

Progress in molecular electrochemical approaches for catalytic reduction of protons to hydrogen is reported in "Complexes of earth-abundant metals for catalytic electrochemical hydrogen generation under aqueous conditions" by Thoi, Sun, Long and Chang (DOI: 10.1039/C2CS35272A). The authors focus on complexes of earth-abundant metals that can function in pure aqueous or mixed aqueous-organic media.

Water-oxidation catalysts (WOCs) featuring a reactive set of four multiredox transition metals are of particular interest because of their close structural and functional relationship to the oxygen evolving complex of natural photosynthesis. In “Tetrametallic molecular catalysts for photochemical water oxidation", Sartorel, Bonchio, Campagna and Scandola (DOI: 10.1039/C2CS35287G) review two classes of tetrametallic molecular WOCs: (i) $\mathrm{M}_{4} \mathrm{O}_{4}$ cubane-type structures stabilized by simple organic ligands, and (ii) systems in which a tetranuclear metal core is stabilized by coordination of two polyoxometalate (POM) ligands.

In their tutorial "Structure-function analyses of solar fuels catalysts using in situ X-ray scattering”, Mulfort, Mukherjee, Kokhan, Du and Tiede (DOI: 10.1039/C2CS35247H) discuss opportunities for the resolution of structurefunction relationships to aid in the development of new materials for solar energy conversion using a combination of spectroscopy and catalysis. They highlight the need to develop new modular, hierarchical, self-healing supramolecular architectures for solar fuels catalysis.

In a theoretical perspective "New concepts and modeling strategies to design and evaluate photo-electro-catalysts based on transition metal oxides", Liao and Carter (DOI: 10.1039/C2CS35267B) argue that theory and computational tools are now sufficiently powerful that it is possible to sort out underlying mechanisms of transition metal oxide semiconductor photocatalysts. They identify accurate theoretical models for improving light absorption, electron-hole transport, band edge alignments of semiconductors, and surface chemistry.

Oh and Hu (DOI: 10.1039/C2CS35276A) discuss the development of "Organic molecules as mediators and catalysts for photocatalytic and electrocatalytic
$\mathrm{CO}_{2}$ reduction". Four classes of organic molecules: tetraalkylammonium salts, aromatic esters and nitriles, ionic liquids, and pyridinium derivatives are found to be competitive compared to their metallo counterparts, both in terms of product selectivity and energy efficiency.

In "Catalysis of the electrochemical reduction of carbon dioxide", Costentin, Robert and Savéant (DOI: 10.1039/ C2CS35360A) present recent findings including the favorable role of acid addition in homogeneous catalytic systems and the crucial chemical role of the electrode material in heterogeneous catalysis.

Ping, Rocca and Galli (DOI: 10.1039/ C3CS00007A) discuss "Electronic excitations in light absorbers for photoelectrochemical energy conversion: first principles calculations based on many body perturbation theory" and some applications of these methods to the study of band edges and absorption processes in representative materials used as photoelectrodes for water splitting.

The technological, environmental, economic, and social benefits of renewable solar fuels cannot be overstated: every human being on earth would be impacted by the development of sustainable energy resources. 